\title{
Forecasting the need for inclusion of the heating system with application of fuzzy logic methods
}

\author{
Vera Bundikova, ${ }^{1, *}$ \\ ${ }^{1}$ Platov South-Russian State Polytechnic University (NPI), Prosveshcheniya str. 132, Novocherkassk, Russia
}

\begin{abstract}
Currently, when predicting the operating modes of heating systems, only temperature is taken into account. But other factors also influence human perceptions: wind speed, humidity, cloudiness, light. When calculating the heat loss of buildings, these factors are also taken into account, but not when determining the operating modes of heating systems. The article proposes to assess the need to turn on the heating system based on the value of a fuzzy function that simulates the concept of «perceived temperature».
\end{abstract}

\section{Introduction}

The main task of the heating system is to provide comfortable living conditions for people in residential and non-residential premises. The microclimate parameters in a residential area are regulated by a number of documents $[1,2]$, according to which the permissible temperature during the cold season should be within $18-24^{\circ} \mathrm{C}$.

Human sensations of the state of external air are influenced not only by temperature, but also by wind speed (table 1), air humidity (table 2), cloudiness, and illumination, which makes it possible to speak of «perceived temperature» [3].When calculating the heat loss of buildings, these factors are also taken into account, but when determining the working time of the heating system, it is not. The heating period must begin / end on the day specified by the regulations (in the Rostov region according to regional norms - October 25 / April 7) or from the day following the day of the end of the 5-day period during which the average daily outdoor temperature was lower / higher than $8^{\circ} \mathrm{C}$ [2]. In the offseason, the weather can be unstable, with large daily temperature variations, strong winds and prolonged rains. As a result, the average daily temperature for a continuous 5-day period is above $8^{\circ} \mathrm{C}$, but the temperature in residential areas is much lower than $18^{\circ} \mathrm{C}$.

System problems of heat supply (dilapidated heating networks and heat insulation, outdated equipment of boiler houses, consumers' non-payment for consumed heat energy) have been seasonally added in recent years: it gets cold early and sharply in the fall, strong wind, rain, high humidity, frost occur at night, however, the air temperature is variable and for 5 days in a row does not fall on average per day below $8^{\circ} \mathrm{C}$. The air temperature in the rooms decreases to $10^{\circ} \mathrm{C}$, but according to the standards for outdoor temperature there are no grounds for switching on the heating system. Similarly, in the spring at the first warming, utilities turn off the heating system, go to maintenance and repair work. Subsequent frosts and strong wind are no longer grounds for switching on the heating, and technically this is impossible.

\section{Member ship function in fuzzy logic}

Fuzzy logic is based on the use of such revolutions of natural language as «far», «close», «cold», «hot». The range of its application is wide - from household appliances to management of complex industrial processes. Many modern control problems cannot be solved by classical methods due to the great complexity of the mathematical models describing them. But in order to use the theory of fuzziness, mathematical transformations are necessary, allowing one to go from linguistic variables to their numerical analogs $[4,5]$. The characteristic of the fuzzy set is the membership function. Denote by MFc (x) the degree of belonging of an object $\mathrm{x}$ to a fuzzy set $\mathrm{C}$, which is a generalization of the concept of the characteristic function of an ordinary set. Then a fuzzy set $C$ is a set of ordered pairs of the form $C=\{M F c(x) / x\}$, $\operatorname{MFc}(x) \in[0,1]$. The value MFc $(x)=0$ means the absence of belonging to the set, 1 - full membership.

\section{Simulation of the heating system activation mode}

After analyzing the data of the last 5 years, we found that the five-day week with an average daily temperature not higher than $+8^{\circ} \mathrm{C}$ comes much later than the boiler houses are involved in (in the Rostov region). At the same time in the premises the temperature is set below the allowable long before the heating system is turned on. We simulate the criterion to determine the need to turn on the heating system based on the concept of «perceived temperature». The boundaries of the perceived temperature are blurred, which allows the use of concepts and methods of fuzzy logic. 
Table 1. Perceived temperature with regard to air temperature and wind speed.

\begin{tabular}{|c|c|c|c|c|c|c|c|c|c|c|c|c|c|c|c|c|c|c|c|}
\hline \multirow{2}{*}{ Temperature, ${ }^{\circ} \mathrm{C}$} & \multicolumn{19}{|c|}{ Windspeed, $\mathrm{m} / \mathrm{s}$} \\
\hline & 1 & 2 & 3 & 4 & 5 & 6 & 7 & 8 & 9 & 10 & 11 & 12 & 13 & 14 & 15 & 16 & 17 & 18 & 19 \\
\hline+10 & +10 & +9 & +9 & +8 & +7 & +7 & +7 & +6 & +6 & +6 & +6 & +6 & +6 & $\begin{array}{ll}+6 \\
\end{array}$ & +5 & +5 & +5 & +5 & +5 \\
\hline+9 & +9 & +8 & +7 & +7 & +6 & +6 & +6 & +5 & +5 & +5 & +5 & +5 & +4 & $\begin{array}{l}+4 \\
\end{array}$ & +4 & +4 & +4 & +4 & +3 \\
\hline+8 & +8 & +7 & +6 & +6 & +5 & +5 & +4 & +4 & +4 & +4 & +3 & +3 & +3 & +3 & +3 & +2 & +2 & +2 & +2 \\
\hline+7 & +7 & +6 & +5 & +4 & +4 & +3 & +3 & +3 & +3 & +2 & +2 & +2 & +2 & +1 & +1 & +1 & +1 & +1 & +1 \\
\hline+6 & +6 & +5 & +4 & +3 & +3 & +2 & +2 & +2 & +1 & +1 & +1 & 0 & 0 & 0 & 0 & 0 & -1 & -1 & -1 \\
\hline+5 & +5 & +3 & +3 & +2 & +1 & +1 & +1 & 0 & 0 & 0 & -1 & -1 & -1 & -1 & -2 & -2 & -2 & -2 & -2 \\
\hline+4 & +4 & +2 & +1 & +1 & 0 & 0 & -1 & -1 & -2 & -2 & -2 & -2 & -3 & -3 & -3 & -3 & -3 & -4 & -4 \\
\hline+3 & +3 & +1 & 0 & -1 & -1 & -2 & -2 & -2 & -3 & -3 & -3 & -4 & -4 & -4 & -4 & -5 & -5 & -5 & -5 \\
\hline+2 & +1 & 0 & -1 & -2 & -2 & -3 & -3 & -4 & -4 & -4 & -5 & -5 & -5 & -5 & -6 & -6 & -6 & -6 & -6 \\
\hline+1 & 0 & -1 & -2 & -3 & -4 & -4 & -5 & -5 & -5 & -6 & -6 & -6 & -7 & -7 & -7 & -7 & -8 & -8 & -8 \\
\hline 0 & -1 & -3 & -4 & -4 & -5 & -6 & -6 & -6 & -7 & -7 & -7 & -8 & -8 & -8 & -8 & -9 & -9 & -9 & -9 \\
\hline-1 & -2 & -4 & -5 & -6 & -6 & -7 & -7 & -8 & -8 & -8 & -9 & -9 & -9 & -10 & -10 & -10 & -10 & -10 & -11 \\
\hline-2 & -3 & -5 & -6 & -7 & -7 & -8 & -9 & -9 & -9 & -10 & -10 & -10 & -11 & -11 & -11 & -11 & -12 & -12 & -12 \\
\hline-3 & -4 & -6 & -7 & -8 & -9 & -9 & -10 & -10 & -11 & -11 & -11 & -12 & -12 & -12 & -13 & $\begin{array}{l}-13 \\
\end{array}$ & $\begin{array}{l}-13 \\
\end{array}$ & $\begin{array}{l}-13 \\
\end{array}$ & -13 \\
\hline-4 & -5 & -7 & $\begin{array}{l}-8 \\
\end{array}$ & -9 & -10 & -11 & -11 & -12 & -12 & -12 & -13 & -13 & -13 & -14 & -14 & -14 & -14 & -15 & -15 \\
\hline-5 & -6 & -8 & -10 & -10 & -11 & -12 & -12 & -13 & -13 & -14 & -14 & -14 & -15 & -15 & -15 & -16 & -16 & -16 & -16 \\
\hline-6 & -8 & -10 & -11 & -12 & -12 & -13 & -14 & -14 & -15 & -15 & -15 & -16 & -16 & -16 & -17 & -17 & -17 & -17 & -18 \\
\hline-7 & -9 & -11 & -12 & -13 & -14 & -14 & -15 & -15 & -16 & -16 & -17 & -17 & -17 & -18 & -18 & -18 & -19 & -19 & -19 \\
\hline-8 & -10 & -12 & -13 & -14 & -15 & -16 & -16 & -17 & -17 & -18 & -18 & -18 & -19 & -19 & -19 & -20 & -20 & -20 & -20 \\
\hline-9 & -11 & -13 & -14 & -15 & -16 & -17 & -18 & -18 & -19 & -19 & -19 & -20 & -20 & -20 & -21 & -21 & -21 & -22 & -22 \\
\hline-10 & -12 & -14 & -16 & -17 & -17 & -18 & -19 & -19 & -20 & -20 & -21 & -21 & -22 & -22 & -22 & -22 & -23 & -23 & -23 \\
\hline-11 & -13 & -15 & -17 & -18 & -19 & -19 & -20 & -21 & -21 & -22 & -22 & -23 & -23 & -23 & -24 & -24 & -24 & -24 & -25 \\
\hline-12 & -14 & -17 & -18 & -19 & -20 & -21 & -21 & -22 & -23 & -23 & -23 & -24 & -24 & -25 & -25 & -25 & -26 & -26 & -26 \\
\hline-13 & -15 & -18 & -19 & -20 & -21 & -22 & -23 & -23 & -24 & -24 & -25 & -25 & -26 & -26 & -26 & -27 & -27 & -27 & -27 \\
\hline-14 & -16 & -19 & -20 & -22 & -23 & -23 & -24 & -25 & -25 & -26 & -26 & -27 & -27 & -27 & -28 & -28 & -28 & -29 & -29 \\
\hline-15 & -18 & -20 & -22 & -23 & -24 & -25 & -25 & -26 & -26 & -27 & -27 & -28 & -28 & -29 & -29 & -29 & -30 & -30 & -30 \\
\hline-16 & -19 & -21 & -23 & -24 & -25 & -26 & -27 & -27 & -28 & -28 & -29 & -29 & -30 & -30 & -30 & -31 & -31 & -31 & -32 \\
\hline-17 & -20 & -22 & -24 & -25 & -26 & -27 & -28 & -29 & -29 & -30 & -30 & -31 & -3 & -31 & -32 & -32 & -32 & -33 & -33 \\
\hline-18 & -21 & -23 & -25 & -26 & -28 & -28 & -29 & -30 & -30 & -31 & -31 & -32 & -32 & -33 & -33 & -34 & -34 & -34 & -35 \\
\hline-19 & -22 & -25 & -26 & -28 & -29 & -30 & -30 & -31 & -32 & -32 & -33 & -33 & -34 & -34 & -35 & -35 & -35 & -36 & -36 \\
\hline-20 & -23 & -26 & -28 & -29 & -30 & -31 & -32 & -32 & -33 & -34 & -34 & -35 & -35 & -35 & -36 & -36 & $\begin{array}{l}-37 \\
\end{array}$ & $\begin{array}{l}-37 \\
\end{array}$ & -37 \\
\hline
\end{tabular}

Table 2. The dependence of the perceived temperature of the relative humidity.

\begin{tabular}{|c|c|c|c|c|c|c|c|c|c|c|c|c|c|c|}
\hline \multirow{2}{*}{ Temperature, ${ }^{\circ} \mathrm{C}$} & \multicolumn{14}{|c|}{ Relative humidity, $\%$} \\
\hline & 30 & 35 & 40 & 45 & 50 & 55 & 60 & 65 & 70 & 75 & 80 & 85 & 90 & 95 \\
\hline-10 & $-23,2$ & $-21,8$ & $-20,4$ & $-19,0$ & $-17,8$ & $-16,7$ & $-15,8$ & $-14,9$ & $-14,1$ & $-13,3$ & $-12,6$ & $-11,9$ & $-10,6$ & $-10,0$ \\
\hline-5 & $-18,9$ & $-17,2$ & $-15,8$ & $-14,5$ & $-13,3$ & $-11,9$ & -109 & $-10,2$ & $-9,3$ & $-8,8$ & $-8,1$ & $-7,7$ & $-6,5$ & $-5,8$ \\
\hline 0 & $-14,5$ & $-12,8$ & $-11,3$ & $-9,9$ & $-8,7$ & $-7,5$ & $-6,2$ & $-5,3$ & $-4,4$ & $-3,5$ & $-2,8$ & $-2,0$ & $-1,3$ & $-0,7$ \\
\hline+2 & $-12,8$ & $-11,0$ & $-9,5$ & $-8,1$ & $-6,8$ & $-5,8$ & $-4,7$ & $-3,6$ & $-2,6$ & $-1,7$ & $-1,0$ & $-0,2$ & $+0,6$ & $+1,3$ \\
\hline+4 & $-11,3$ & $-9,5$ & $-7,9$ & $-6,5$ & $-4,9$ & $-4,0$ & $-3,0$ & $-1,9$ & $-1,0$ & $+0,0$ & $+0,8$ & $+1,6$ & $+2,4$ & $+3,2$ \\
\hline+5 & $-10,5$ & $-8,7$ & $-7,3$ & $-5,7$ & $-4,3$ & $-3,3$ & $-2,2$ & $-1,1$ & $-0,1$ & $+0,7$ & $+1,6$ & $+2,5$ & $+3,3$ & $+4,1$ \\
\hline+6 & $-9,5$ & $-7,7$ & $-6,0$ & $-4,5$ & $-3,3$ & $-2,3$ & $-1,1$ & $-0,1$ & $+0,8$ & $+1,8$ & $+2,7$ & $+3,6$ & $+4,5$ & $+5,3$ \\
\hline+7 & $-9,0$ & $-7,2$ & $-5,5$ & $-4,0$ & $-2,8$ & $-1,5$ & $-0,5$ & $+0,7$ & $+1,6$ & $+2,5$ & $+3,4$ & $+4,3$ & $+5,2$ & $+6,1$ \\
\hline+8 & $-8,2$ & -6 , & $-4,7$ & $-3,3$ & $-2,1$ & $-0,9$ & $+0,3$ & $+1,3$ & $+2,3$ & $+3,4$ & $+4,5$ & $+5,4$ & $+6,2$ & $+7,1$ \\
\hline+9 & $-7,5$ & $-5,5$ & $-3,9$ & $-2,5$ & $-1,2$ & $+0,0$ & $+1,2$ & $+2,4$ & $+3,4$ & $+4,5$ & $+5,5$ & $+6,4$ & $+7,3$ & $+8,2$ \\
\hline+10 & $-6,7$ & $-5,2$ & $-3,2$ & $-1,7$ & $-0,3$ & $+0,8$ & $+2,2$ & $+3,2$ & $+4,4$ & $+5,5$ & $+6,4$ & $+7,3$ & $+8,2$ & $+9,1$ \\
\hline+11 & $-6,0$ & $-4,0$ & $-2,4$ & $-0,9$ & $+0,5$ & $+1,8$ & $+3,0$ & $+4,2$ & $+5,3$ & $+6,3$ & $+7,4$ & $+8,3$ & $\begin{array}{r}+9,2 \\
\end{array}$ & $+10,1$ \\
\hline+12 & $-4,9$ & $-3,3$ & $-1,6$ & $-0,1$ & $+1,6$ & $+2,8$ & $+4,1$ & $+5,2$ & $+6,3$ & $+7,5$ & $+8,6$ & $+9,5$ & $+10,4$ & $+11,7$ \\
\hline+13 & $-4,3$ & $-2,5$ & $-0,7$ & $+0,7$ & $+2,2$ & $+3,6$ & $+5,2$ & $+6,4$ & $+7,5$ & $+8,4$ & $+9,5$ & $+10,5$ & $+11,5$ & $+12,3$ \\
\hline $\begin{array}{l}+14 \\
\end{array}$ & $-3,7$ & $-1,7$ & $-0,0$ & $\begin{array}{r}+1,5 \\
\end{array}$ & $+3,0$ & $+4,5$ & $\begin{array}{l}+5,8 \\
\end{array}$ & $+7,0$ & $\begin{array}{l}+8,2 \\
\end{array}$ & $\begin{array}{r}+9,3 \\
\end{array}$ & $+10,3$ & $+11,2$ & $+12,1$ & $+13,1$ \\
\hline+15 & $-2,9$ & $-1,0$ & $+0,8$ & $+2,4$ & $+4,0$ & $+5,5$ & $+6,7$ & $+8,0$ & $+9,2$ & $+10,2$ & $+11,2$ & $+12,2$ & $+13,1$ & $+14,1$ \\
\hline+16 & $-2,1$ & $-0,1$ & $+1,5$ & $+3,2$ & $+5,0$ & $+6,3$ & $+7,6$ & $+9,0$ & $+10,2$ & $+11,3$ & $+12,2$ & $+13,2$ & $+14,2$ & $+15,1$ \\
\hline+17 & $-1,3$ & $+0,6$ & $+2,5$ & $+4,3$ & $+5,9$ & $+7,2$ & $+8,8$ & $+10,0$ & $+11,2$ & $+12,2$ & $+13,5$ & $+14,3$ & $+15,2$ & $+16,6$ \\
\hline+18 & $-0,5$ & $+1,5$ & $+3,2$ & $+5,3$ & $+6,8$ & $+8,2$ & $+9,6$ & $+11,0$ & $+12,2$ & $+13,2$ & $+14,2$ & $+15,3$ & $+16,2$ & $+17,1$ \\
\hline+19 & $+0,3$ & $+2,2$ & $+4,2$ & $+6,0$ & $+7,7$ & $+9,2$ & $+10,5$ & $+11,7$ & $+13,0$ & $+14,2$ & $+15,2$ & $+16,3$ & $+17,2$ & $+18,1$ \\
\hline+20 & $+1,0$ & $+3,1$ & $+5,2$ & $+7,0$ & $+8,7$ & $+11,5$ & $+11,5$ & $+12,8$ & $+14,0$ & $+15,2$ & $+16,2$ & $+17,2$ & $+18,1$ & $+19,1$ \\
\hline+21 & $+1,8$ & $+4,0$ & $\begin{array}{r}+6,0 \\
+6,0\end{array}$ & $\begin{array}{r}+7,9 \\
\end{array}$ & $+9,5$ & $+11,1$ & $+12,4$ & $+13,5$ & $+15,0$ & $+16,2$ & $+17,2$ & $+18,1$ & $+19,1$ & $+20,0$ \\
\hline+22 & $+2,5$ & $+5,0$ & $+6,9$ & $+8,8$ & $+10,5$ & $+11,9$ & $+13,5$ & $+14,8$ & $+16,0$ & $+17,0$ & $+18,0$ & $+19,0$ & $+20,0$ & $+21,0$ \\
\hline+23 & $+3,5$ & $+5,7$ & $+7,8$ & $+9,8$ & $+11,5$ & $+12,9$ & $+14,3$ & $+15,7$ & $+16,9$ & $+18,1$ & $+19,1$ & $+20,0$ & $+21,0$ & $+22,0$ \\
\hline+24 & $+4,3$ & $+6,7$ & $+8,8$ & $+10,8$ & $+12,3$ & $+13,8$ & $+15,3$ & $+16,5$ & $+17,8$ & $+19,0$ & $+20,1$ & $+21,1$ & $+22,0$ & $+23,0$ \\
\hline+25 & $+5,2$ & $+7,5$ & $+9,7$ & $+11,5$ & $+13,1$ & $+14,7$ & $+16,2$ & $+17,5$ & $+18,8$ & $+20,0$ & $+21,1$ & $+22,1$ & $+23,0$ & $+24,0$ \\
\hline+26 & $+6,0$ & $+8,5$ & $+10,6$ & $+12,4$ & $+14,2$ & $+15,8$ & $+17,2$ & $+18,5$ & $+19,8$ & $+21,0$ & $+22,2$ & $+24,1$ & $+25,0$ & $+26,1$ \\
\hline+27 & $+6,9$ & $+9,5$ & $+11,4$ & $+13,3$ & $+15,2$ & $+16,5$ & $\begin{array}{l}+18,1 \\
\end{array}$ & $+19,5$ & $+20,7$ & $+21,9$ & $+23,1$ & $+24,1$ & $+25,0$ & $26,1+$ \\
\hline+28 & $+7,7$ & $+10,2$ & $+12,2$ & $+14,2$ & $+16,0$ & $+17,5$ & $+19,0$ & $+20,5$ & $+21,7$ & $+21,7$ & $+24,0$ & $+25,1$ & $+26,1$ & $+27,0$ \\
\hline $\begin{array}{r}+29 \\
\end{array}$ & $\begin{array}{r}+8,7 \\
\end{array}$ & $+11,1$ & $+13,1$ & $+15,1$ & $+16,8$ & $+18,5$ & $\begin{array}{r}+19,9 \\
\end{array}$ & $+21,3$ & $+22,5$ & $+22,8$ & $+25,0$ & $+26,0$ & $+27,0$ & $+28,0$ \\
\hline+30 & $+9,5$ & $+11,8$ & $+13,9$ & $+16,0$ & $+17,7$ & $+19,7$ & $+21,3$ & $+22,5$ & $+23,8$ & $+25,0$ & $+26,1$ & $+27,1$ & $+28,1$ & $+29,0$ \\
\hline+32 & $+11,2$ & $+13,8$ & $+16,0$ & $+17,9$ & $+19,7$ & $+21,4$ & $+22,8$ & $+24,3$ & $+25,6$ & $+26,7$ & $+28,0$ & $+29,2$ & $+30,2$ & $+31,1$ \\
\hline+34 & $+12,5$ & $+15,2$ & $+17,2$ & $+19,2$ & $+21,4$ & $+22,8$ & $+24,2$ & $+25,7$ & $+27,0$ & $+28,3$ & $+29,4$ & $+31,1$ & $+31,9$ & $+33,0$ \\
\hline+36 & $+14,6$ & $+17,1$ & $+19,4$ & $+21,5$ & $+23,2$ & $+25,0$ & $+26,3$ & $+28,0$ & $+29,3$ & $+30,7$ & $+31,8$ & $+32,8$ & $+34,0$ & $+35,1$ \\
\hline $\begin{array}{r}+38 \\
\end{array}$ & $+16,3$ & $+18,8$ & $+21,3$ & $+23,4$ & $+25,1$ & $+26,7$ & $+28,3$ & $\begin{array}{l}+29,9 \\
\end{array}$ & $+31,2$ & $+32,3$ & $+33,5$ & $+34,6$ & $+35,7$ & $+36,9$ \\
\hline
\end{tabular}

According to the table 1 , at $+14^{\circ} \mathrm{C}$ and wind speed of $19 \mathrm{~m} / \mathrm{s}$, the temperature is felt as $+8^{\circ} \mathrm{C}$. Therefore, in this case, to ensure the quality of heat supply, it is necessary to start the heating season from $+14^{\circ} \mathrm{C}$. We assume that heating is definitely not included at $+15^{\circ} \mathrm{C}$ and at any wind, and $a t+8^{\circ} \mathrm{C}$ it is also unambiguously included at any 
wind. We write this in the form of mathematical dependence:

$$
\frac{\mathrm{t}-\mathrm{t}_{1}}{\mathrm{t}_{2}-\mathrm{t}_{1}}=\frac{\mathrm{P}-\mathrm{P}_{1}}{\mathrm{P}_{2}-\mathrm{P}_{1}} .
$$

where $(\mathrm{t} 1, \mathrm{P} 1)=(8,1),(\mathrm{t} 2, \mathrm{P} 2)=(15,0)$, then

$$
\frac{\mathrm{t}-8}{15-8}=\frac{\mathrm{P}-1}{0-1} \text { or } P=1-\frac{\mathrm{t}-8}{7} \text {. }
$$

Get

$$
P_{t}=\left\{\begin{array}{cc}
1, & t \leq 8 \\
1-\frac{(t-8)}{7}, & 8<t<15, \\
0, & t \geq 15
\end{array}\right.
$$

where $\mathrm{Pt}$ is the characteristic of belonging to the fuzzy set "Include heating system». Pt $=1$ means that the heating system should be turned on immediately, $\mathrm{Pt}=0$ means that the heating system should not be turned on as unequivocally, $\mathrm{Pt}>0.5$ - the heating system should be turned on rather than not turned on (fig. 1).

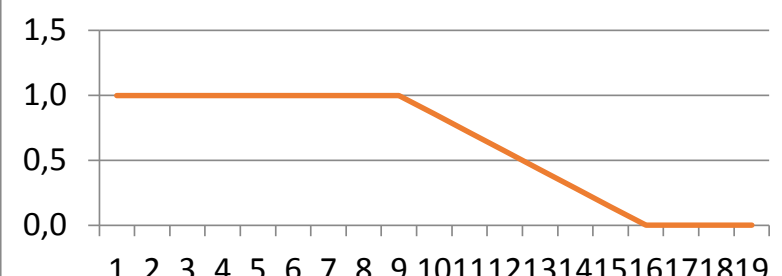

Fig. 1. The graph of the dependence of belonging function to the fuzzy set «Include heating system» on the temperature.

Since the heating system cannot be in the «partially on» state and taking into account the weather conditions, correlation (1) must be amended.

Wind speed correction. For wind speed, where

$$
(\mathrm{V} 1, \mathrm{P} 1)=(19,1),(\mathrm{V} 2, \mathrm{P} 2)=(2,0), \text { get }(\text { fig. } 2) \text { : }
$$

$$
\begin{gathered}
\frac{\mathrm{V}-19}{2-19}=\frac{\mathrm{P}-1}{0-1} \text { or } P=1+\frac{\mathrm{V}-19}{17}, \\
P_{V}=\left\{\begin{array}{cc}
1, & V \geq 2 \\
1+\frac{(V-19)}{17}, & 2<V<19, \\
0, & V \leq 19
\end{array}\right.
\end{gathered}
$$

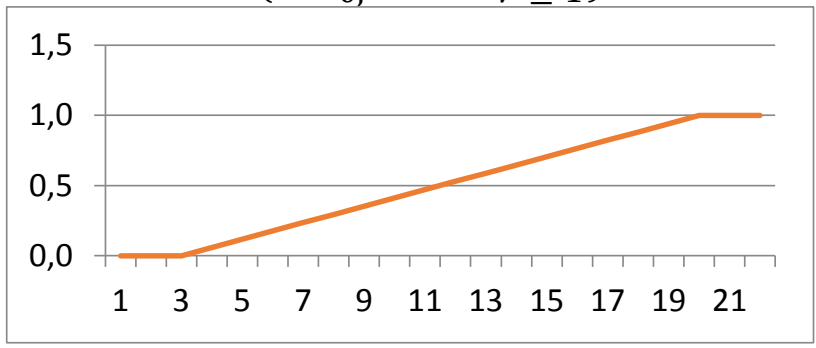

Fig. 2. The graph of the dependence of amendment to the belonging function to the set «Include heating system» on the wind speed.

Correction for humidity. For relative air humidity, where

$(\mathrm{w} 1, \mathrm{P} 1)=(90,1),(\mathrm{w} 2, \mathrm{P} 2)=(95,0)$, we obtain $($ fig. 3$)$ :

$$
\frac{\mathrm{w}-90}{95-90}=\frac{\mathrm{P}-1}{0-1} \text { or } P=1-\frac{\mathrm{w}-90}{5},
$$

$$
P_{w}=\left\{\begin{array}{cc}
1, & w \leq 90 \\
1-\frac{(w-90)}{5}, & 90<w<95 \\
0, & w \geq 95
\end{array}\right.
$$

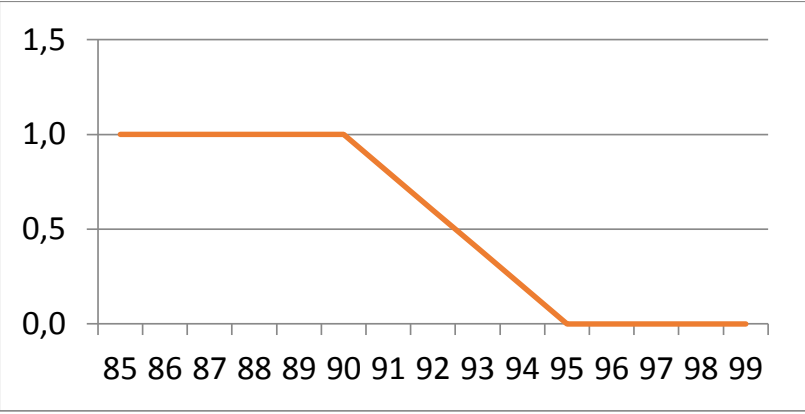

Fig. 3. The graph of the dependence of amendment to the belonging function to the set «Include heating system» on the relative humidity of air.

We write the value of the belonging function (1) with the amendments (2), (3) and restrictions:

$$
\begin{gathered}
V_{O}=\left\{\begin{array}{cc}
2, & V \leq 2 \\
V, & 2<V<19, \\
19, & V \geq 19
\end{array}\right. \\
W_{O}=\left\{\begin{array}{cc}
90, & w \leq 90 \\
w, & 90<w<95, \\
95, & w \geq 95
\end{array}\right.
\end{gathered}
$$

$P_{0}(t, V, W)=\min \left\{1 ;\left(\left(1-\frac{(t-8)}{7}\right)+\frac{V-2}{17}+\frac{95-W}{5}\right)\right\}$,

Obtain:

$$
P_{t, V, W}=\left\{\begin{array}{lr}
1, & t \leq 8 \\
P_{0}(t, V, W), & 8<t<15 \\
P_{0}(15, V, W)\left(1-\frac{t-15}{3}\right), & 15 \leq t<18 \\
0, & t \geq 18
\end{array},\right.
$$

Thus, the final membership function $\mathrm{P}_{\mathrm{t}, \mathrm{V}, \mathrm{W}}$ (7) allows to take into account the state of the outside air, as soon as its temperature falls below $+18^{\circ} \mathrm{C}-$ the minimum allowable temperature for rooms according to standards, while taking into account wind speed and humidity. This characteristic better reflects the totality of natural factors affecting the temperature sensed by man. In addition, at high wind speeds, the rooms are faster cooled, and high humidity is present not only in the outside air, but also in unheated rooms.

\section{Calculation examples}

The function of $\mathrm{P}_{\mathrm{t}, \mathrm{V}, \mathrm{W}}$ can reach a value of 1 at temperatures above $8^{\circ} \mathrm{C}$. According to (6), (7), at a wind speed of $19 \mathrm{~m} / \mathrm{s}$ and more, with a humidity of $90 \%$ or less, the heating system must be switched on already at $15^{\circ} \mathrm{C}$. Such winds happen (in the Rostov region) infrequently, the humidity in rainy weather is close to $100 \%$. Consider intermediate values.

At $\mathrm{t}=10^{\circ} \mathrm{C}, \mathrm{V}=7 \mathrm{~m} / \mathrm{s}, \mathrm{W}=99 \%$, we get

$$
P_{t, V, W}=P_{0}(t, V, W)=
$$




$$
\begin{gathered}
\min \left\{1 ;\left(\left(1-\frac{(10-8)}{7}\right)+\frac{7-2}{17}+\frac{95-95}{5}\right)\right\}= \\
\min \{1 ; 1,0084\}=1
\end{gathered}
$$

that is, the heating system must be immediately turned on.

At $\mathrm{t}=9^{\circ} \mathrm{C}, \mathrm{V}=3 \mathrm{~m} / \mathrm{s}, \mathrm{W}=94 \%$, we get

$$
\begin{gathered}
P_{t, V, W}=P_{0}(t, V, W)= \\
\min \left\{1 ;\left(\left(1-\frac{(9-8)}{7}\right)+\frac{3-2}{17}+\frac{95-94}{5}\right)\right\}= \\
\min \{1 ; 1,1160\}=1,
\end{gathered}
$$

the heating system must also be turned on. However, if rainy weather sets in at this temperature and wind $(\mathrm{W}=$ $100 \%$ ), then

$$
\begin{gathered}
P_{t, V, W}=P_{0}(t, V, W)= \\
\min \left\{1 ;\left(\left(1-\frac{(9-8)}{7}\right)+\frac{3-2}{17}+\frac{95-95}{5}\right)\right\}= \\
\min \{1 ; 0,9160\}=0,916,
\end{gathered}
$$

that is, the heating system should be switched on rather than not turned on.

\section{Conclusion}

The use of fuzzy logic methods makes it possible to more objectively assess the need to turn on the heating system for various combinations of weather factors.

\section{References}

1. State standard 30494-2011. Residential and public buildings. The parameters of the microclimate in the premises (2011)

2. Set of rules 131.13330.2012. Construction climatology (2012)

3. Climate data of cities around the world URL: https://ru.climate-data.org/

4. L. Zade, The concept of a linguistic variable and its application to making approximate decisions (World, Moscow, 1976)

5. Base GRUP Labs: Data Analysis Technologies URL: https://basegroup.ru/ 